\title{
COVID-19 Incidence and Mortality Among American Indian/Alaska Native and White Persons - Montana, March 13-November 30, 2020
}

\author{
Laura L. Williamson, $\mathrm{MPH}^{1}$; Todd S. Harwell, $\mathrm{MPH}^{1}$; Todd M. Koch, MPH ${ }^{1}$; Stacey L. Anderson, $\mathrm{MPH}^{1}$; Magdalena K. Scott, MPH ${ }^{1}$; \\ James S. Murphy ${ }^{1}$; Greg S. Holzman, MD ${ }^{1}$; Helen F. Tesfai, $\mathrm{MPH}^{2}$
}

Geographic differences in infectious disease mortality rates have been observed among American Indian or Alaska Native (AI/AN) persons in the United States (1), and aggregate analyses of data from selected U.S. states indicate that COVID-19 incidence and mortality are higher among AI/AN persons than they are among White persons $(2,3)$. State-level data could be used to identify disparities and guide local efforts to reduce COVID-19-associated incidence and mortality; however, such data are limited. Reports of laboratory-confirmed COVID-19 cases and COVID-19-associated deaths reported to the Montana Department of Public Health and Human Services (MDPHHS) were analyzed to describe COVID-19 incidence, mortality, and case-fatality rates among AI/AN persons compared with those among White persons. During March-November 2020 in Montana, the estimated cumulative COVID-19 incidence among AI/AN persons (9,064 cases per 100,000) was 2.2 times that among White persons $(4,033$ cases per 100,000$)$.* During the same period, the cumulative COVID-19 mortality rate among AI/AN persons (267 deaths per 100,000) was 3.8 times that among White persons (71 deaths per 100,000). The AI/AN COVID-19 case-fatality rate (29.4 deaths per 1,000 COVID-19 cases) was 1.7 times the rate in White persons (17.0 deaths per 1,000). State-level surveillance findings can help in developing state and tribal COVID-19 vaccine allocation strategies and assist in local implementation of culturally appropriate public health measures that might help reduce COVID-19 incidence and mortality in AI/AN communities.

Reports of COVID-19 cases and COVID-19-associated deaths were analyzed to assess the number, percentage, and crude rates of COVID-19 cases and deaths occurring among AI/AN persons and White persons in Montana during March 13-November 30, 2020. Case data were derived from the Montana Infectious Disease Information System. Montana residents who met the definition of a confirmed case (i.e., having received a positive test result for SARS-CoV-2, the virus that causes COVID-19, from a respiratory specimen, using a molecular amplification test and reported to MDPHHS) were

\footnotetext{
* Race data were missing for 13,913 of 63,339 (22\%) patients, and ethnicity data were missing for 23,435 of 63,339 (37\%) patients. Race and ethnicity data were complete for all deaths. Cumulative incidences by race were considered estimates because of the large proportion of missing race/ethnicity data.
}

included in the analysis. COVID-19-associated deaths were identified from death certificates reported to the MDPHHS Office of Vital Records; COVID-19 deaths were identified by using International Classification of Diseases, Tenth Revision code U07.1, listed as either the underlying or a contributing cause of death. This activity was reviewed by MDPHHS and was conducted for public health surveillance purposes and consistent with applicable state and federal law. ${ }^{\dagger}$

Information on race was available for $49,426(78 \%)$ of 63,339 persons who had received a diagnosis of COVID-19 and for 903 (100\%) COVID-19-associated deaths reported to MDPHHS. Persons of AI/AN race were defined as those whose race was reported as $\mathrm{AI} / \mathrm{AN}$ alone or in combination with other races. Persons of White race were defined as those whose race was reported as White with no other race selected. Ethnicity was not included in this analysis because data on ethnicity was missing for 37\% of reported cases. The 2019 National Center for Health Statistics bridged-race population estimates for AI/AN and White persons in Montana were used as denominators to calculate crude cumulative incidence (cumulative cases per 100,000 population) and cumulative mortality rates (cumulative deaths per 100,000 population). $\$, \Phi$ These population estimates were used to determine that $90.6 \%$ of Montana residents were White and that $7.3 \%$ were AI/AN. All rates were calculated separately for AI/AN and White persons, overall and by sex and age group. Age group was assessed both categorically ( $<65$ and $\geq 65$ years) and by using medians with interquartile ranges (IQRs). Rate ratios (COVID-19 cumulative incidence and mortality rates among AI/AN persons divided by corresponding rates among White persons) and case-fatality rates (the number of COVID-19-associated deaths per 1,000 reported COVID-19 cases) were also calculated. Corresponding $95 \%$ confidence intervals (CIs) were calculated using the Poisson Exact method (4). Analyses were conducted using SPSS (version 23; IBM) for incidence estimates and SAS (version 9.4; SAS Institute) for mortality estimates.

\footnotetext{
${ }_{\dagger}$ Mont. Code Ann. Sect. 50-15-122 and 50-16-103; 45 C.F.R. part 46.102(1)(2). $\$$ https://www.cdc.gov/nchs/nvss/bridged_race/data_documentation.htm

The number of COVID-19 cases or deaths by race and by race and sex/age group per 100,000 in the same race or race and sex/age group.
} 
During March 13-November 30, 2020, among 49,426 persons in Montana who had received a diagnosis of COVID-19 and for whom information on race was available, 7,069 (14.3\%) were AI/AN, and 39,040 (79.0\%) were White. The estimated cumulative incidence among AI/AN persons $(9,064$ cases per 100,000$)$ was 2.2 times the rate among White persons $(4,033)$ (Table). The estimated cumulative incidence was also higher among AI/AN persons than that among White persons by sex and age group. Among AI/AN persons, the estimated cumulative incidence was higher among persons aged $\geq 65$ years $(10,321$ per 100,000$)$ compared with that among persons aged $<65$ years $(8,947)$. ${ }^{* *}$ Among White persons, incidence was higher among persons aged $<65$ years $(4,137)$ than among those aged $\geq 65$ years $(3,632)$. The median age of AI/AN persons with COVID-19 was 34 years (IQR $=20-51$ years) compared with 42 years (IQR = 26-60 years) among White persons. Among both AI/AN and White persons, estimated cumulative incidence by race was higher among women $(9,517$ and 4,272 per 100,000 , respectively) than among men $(8,405$ and 3,687, respectively).

During March 13-November 30, 2020, among 903 COVID-19-associated deaths in Montana, 208 (23.0\%) occurred among AI/AN persons compared with 664 (73.5\%)

\footnotetext{
** A total of 681 cases of COVID-19 were reported among 6,598 AI/AN persons aged $\geq 65$ years, and 6,388 cases were reported among $71,395 \mathrm{AI} / \mathrm{AN}$ persons aged $<65$ years.
}

among White persons. The cumulative COVID-19 mortality rate among AI/AN persons (267 deaths per 100,000) was 3.8 times the rate among White persons (71 deaths per $100,000)$. Cumulative mortality was also higher among AI/AN persons than among White persons by sex and age group. The median age of death among AI/AN persons who died from COVID-19 was 68 years (IQR $=58-75)$ compared with 82 years $(\mathrm{IQR}=73-89)$ among White persons. The casefatality rate among AI/AN persons (29.4 deaths per 1,000 COVID-19 cases) was 1.7 times $(95 \% \mathrm{CI}=1.7-1.8)$ the rate among White persons (17.0 deaths per 1,000 COVID-19 cases).

\section{Discussion}

During March 13-November 30, 2020, COVID-19 incidence and mortality among AI/AN persons in Montana were approximately twice and nearly four times those among White persons, respectively. In addition, the case-fatality rate among AI/AN persons was close to twice that among White persons. Several factors might have contributed to the higher COVID-19 incidence and mortality among AI/AN persons. AI/AN communities in Montana have higher levels of social vulnerability, including living in shared housing, challenges accessing health care and transportation, and lower household incomes. ${ }^{\dagger \dagger}$ As well, AI/AN persons might be more likely than

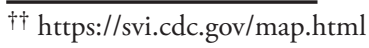

TABLE. COVID-19 incidence and mortality rates* among American Indian or Alaska Native (AI/AN) and White persons, sex" - Montana, March 13-November 30, 2020

\begin{tabular}{|c|c|c|c|c|c|}
\hline \multirow[b]{2}{*}{ Characteristic } & \multicolumn{2}{|c|}{$\mathrm{Al} / \mathrm{AN}$} & \multicolumn{2}{|c|}{ White } & \multirow{2}{*}{$\begin{array}{c}\mathrm{Al} / \mathrm{AN} \text { to White rate ratio } \\
(95 \% \mathrm{Cl})\end{array}$} \\
\hline & No. (\%) & Rate $(95 \% \mathrm{Cl})$ & No. (\%) & Rate $(95 \% \mathrm{Cl})$ & \\
\hline \multicolumn{6}{|c|}{ Cumulative incidence } \\
\hline Total & $7,069(100)$ & $9,064(8,852-9,275)$ & $39,040(100)$ & $4,033(3,993-4,073)$ & $2.2(2.1-2.5)$ \\
\hline $\begin{array}{l}\text { Sex } \\
\text { Female } \\
\text { Male }\end{array}$ & $\begin{array}{l}3,752(53) \\
3,242(46)\end{array}$ & $\begin{array}{l}9,517(9,212-9,821) \\
8,405(8,116-8,695)\end{array}$ & $\begin{array}{l}20,498(52) \\
17,995(46)\end{array}$ & $\begin{array}{l}4,272(4,213-4,330) \\
3,687(2,633-3,741)\end{array}$ & $\begin{array}{l}2.2(2.1-2.4) \\
2.3(2.1-2.5)\end{array}$ \\
\hline $\begin{array}{l}\text { Age group, yrs } \\
<65 \\
\geq 65\end{array}$ & $\begin{array}{r}6,388(90) \\
681(10)\end{array}$ & $\begin{array}{r}8,947(8,728-9,167) \\
10,321(9,546-1,097)\end{array}$ & $\begin{array}{r}31,842(82) \\
7,198(18)\end{array}$ & $\begin{array}{l}4,137(4,091-4,182) \\
3,632(3,549-3,716)\end{array}$ & $\begin{array}{l}2.2(2.0-2.4) \\
2.8(2.6-3.1)\end{array}$ \\
\hline Cumulative mo & & & & & \\
\hline Total & $208(100)$ & $267(232-306)$ & $664(100)$ & $71(66-77)$ & $3.8(3.2-4.4)$ \\
\hline $\begin{array}{l}\text { Sex } \\
\text { Female } \\
\text { Male }\end{array}$ & $\begin{array}{r}88(42) \\
120(58)\end{array}$ & $\begin{array}{l}223(179-275) \\
311(258-372)\end{array}$ & $\begin{array}{l}306(46) \\
358(54)\end{array}$ & $\begin{array}{l}66(59-74) \\
76(68-84)\end{array}$ & $\begin{array}{l}3.4(2.7-4.3) \\
4.1(3.3-5.0)\end{array}$ \\
\hline $\begin{array}{l}\text { Age group, yrs } \\
<65 \\
\geq 65\end{array}$ & $\begin{array}{r}87(42) \\
121(58)\end{array}$ & $\begin{array}{r}122(98-150) \\
1,834(1,522-2,191)\end{array}$ & $\begin{array}{r}72(11) \\
592(89)\end{array}$ & $\begin{array}{r}10(8-12) \\
302(278-328)\end{array}$ & $\begin{array}{r}12.5(9.1-17.1) \\
6.1(5.0-7.4)\end{array}$ \\
\hline
\end{tabular}

Abbreviation: $\mathrm{Cl}=$ confidence interval.

* The number of COVID-19 cases or deaths by race and by race and sex/age group per 100,000 in the same race or race and sex/age group.

$\dagger$ Includes Hispanic and non-Hispanic persons.

$\S$ Race data were missing for 13,913 of 63,339 (22\%) patients, and ethnicity data were missing for 23,435 of 63,339 (37\%) patients; race and ethnicity data were complete for all deaths.

I Sex data were missing for 75 (1\%) Al/AN patients and for 547 (1\%) White patients. 
White persons to live in multigenerational households or be unable to work from home because of the nature of their work (e.g., being frontline workers) or because they are not able to telework due to the lack of Internet access, which might increase the risk for SARS-CoV-2 infection (5). AI/AN persons in Montana also have a high prevalence of chronic health conditions and risk factors for severe illness from COVID-19, including heart disease, type 2 diabetes mellitus, and cigarette smoking. ${ }^{\$ S}$

An assessment of the impact of COVID-19 among AI/AN persons from 23 states made early during the pandemic (January-July 2020) found that the cumulative incidence rate ratio between AI/AN and White persons was 3.5 (2), similar to the current study's finding. In addition, a study comparing age-adjusted COVID-19 mortality rates among AI/AN and White persons in 14 states during January-June 2020 reported a mortality rate ratio of 1.8 for AI/AN persons compared with that for White persons (3), which was lower than that identified in a supplementary analysis conducted by MDPHHS. 99

The findings in this report are subject to at least three limitations. First, the case-level surveillance and death certificate data might not have been complete at the time of the analysis and are subject to change. Therefore, this analysis likely underestimated the number of persons who had received a diagnosis of COVID-19 and the number of deaths that occurred, particularly more recently. Second, information on race and ethnicity was missing for $22 \%$ and $37 \%$ of cases, respectively. Because of the large proportion of COVID- 19 cases with missing ethnicity information, ethnicity was not included in the analyses. Therefore, COVID-19 patients and decedents whose race was listed as White include some persons whose ethnicity is Hispanic or non-Hispanic. Previous reports have documented that Hispanic populations have disproportionality higher COVID-19 mortality compared with White populations (G). However, the extent of bias introduced as a result of this limitation is expected to be minimal because Hispanic persons represent a small proportion of Montana's population (4.1\%). Finally, previous studies suggest that AI/AN persons might be misclassified as non-AI/AN races and ethnicities. However, studies conducted in Montana and northern plains states indicate that race misclassification is relatively less common in these states than it is in other areas of the United States $(7,8)$.

Understanding the higher COVID-19 incidence, mortality, and case-fatality rates among AI/AN persons can help develop state and tribal COVID-19 vaccine allocation

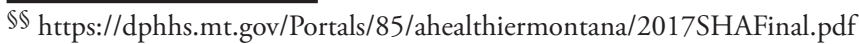

99 The MDPHHS calculated age-adjusted COVID-19 mortality rate for AI/AN persons (374 deaths per 100,000; 95\% CI $=322-433$ ) was 7.8 times $(95 \% \mathrm{CI}=6.7-9.1)$ that among White persons (48 deaths per 100,000; $95 \% \mathrm{CI}=44-52$ ). Rates were age-adjusted to the 2000 U.S. standard population.
}

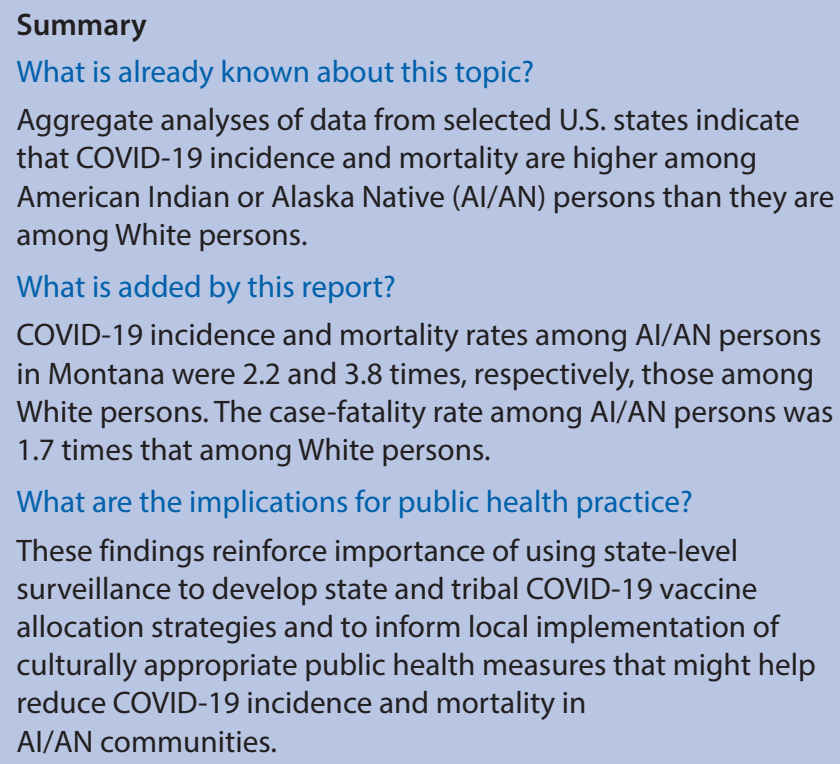

strategies, including adapting the current interim CDC Advisory Committee on Immunization Practices' COVID-19 vaccine allocation recommendations to prioritize persons at increased risk for poor outcomes or at high risk for exposure to SARS-CoV-2 in AI/AN communities, such as tribal elders, persons living in multigenerational or congregate households, and persons with high-risk medical conditions (9). These findings also reinforce the importance of using state-level surveillance to identify disparities among AI/AN or other minority communities to help develop local implementation of culturally informed public health measures and enhanced community education to prevent or limit community transmission of SARS-CoV-2 (10).

\section{Acknowledgments}

State, local, and tribal public health and Indian Health Service personnel; Jeremy Gold, CDC.

Corresponding author: Laura L. Williamson, lwilliamson@mt.gov.

${ }^{1}$ Montana Department of Public Health and Human Services; ${ }^{2}$ Rocky Mountain Tribal Leaders Council, Epidemiology Center, Billings, Montana.

All authors have completed and submitted the International Committee of Medical Journal Editors form for disclosure of potential conflicts of interest. No potential conflicts of interest were disclosed.

\section{References}

1. Groom AV, Hennessy TW, Singleton RJ, Butler JC, Holve S, Cheek JE. Pneumonia and influenza mortality among American Indian and Alaska Native people, 1990-2009. Am J Public Health 2014;104(Suppl3):S460-9. PMID:24754620 https://doi.org/10.2105/AJPH.2013.301740

2. Hatcher SM, Agnew-Brune C, Anderson M, et al. COVID-19 among American Indian and Alaska Native persons-23 states, January 31-July 3, 2020. MMWR Morb Mortal Wkly Rep 2020;69:1166-9. PMID:32853193 https://doi.org/10.15585/mmwr.mm6934e1 
3. Arrazola J, Masiello MM, Joshi S, et al. COVID-19 mortality among American Indian and Alaska Native persons-14 states, January-June 2020. MMWR Morb Mortal Wkly Rep 2020;69:1853-6. PMID:33301432 https://doi.org/10.15585/mmwr.mm6949a3

4. Patil DS, Liu J, Su Z. Comparing confidence intervals of incidence rates. Value Health 2016;19:A99. https://doi.org/10.1016/j.jval.2016.03.1713

5. Fraser-Lee NJ, Hessel PA. Acute respiratory infections in the Canadian Native Indian population: a review. Can J Public Health 1994;85:197-200. PMID:7922966

6. Gold JAW, Rossen LM, Ahmad FB, et al. Race, ethnicity, and age trends in persons who died from COVID-19-United States, May-August 2020. MMWR Morb Mortal Wkly Rep 2020;69:1517-21. PMID:33090984 https://doi.org/10.15585/mmwr.mm6942e1

7. Harwell TS, Hansen D, Moore KR, Jeanotte D, Gohdes D, Helgerson SD. Accuracy of race coding on American Indian death certificates, Montana 1996-1998. Public Health Rep 2002;117:44-9. PMID:12297681 https://doi.org/10.1093/phr/117.1.44
8. Jim MA, Arias E, Seneca DS, et al. Racial misclassification of American Indians and Alaska Natives by Indian Health Service contract health service delivery area. Am J Public Health 2014;104(Suppl 3):S295-302. PMID:24754617 https://doi.org/10.2105/AJPH.2014.301933

9. Dooling K, Marin M, Wallace M, et al. The Advisory Committee on Immunization Practices' updated interim recommendation for allocation of COVID-19 vaccine-United States, December 2020. MMWR Morb Mortal Wkly Rep 2021;69:1657-60. PMID:33382671 https://doi. org/10.15585/mmwr.mm695152e2

10. Hirschman J, Kaur H, Honanie K, et al. A SARS-CoV-2 outbreak illustrating the challenges in limiting the spread of the virus-Hopi tribe, May-June 2020. MMWR Morb Mortal Wkly Rep 2020;69:1654-9. PMID:33151922 https://doi.org/10.15585/mmwr.mm6944a5 